\title{
Slow motor neuron stimulation of locust skeletal muscle: model and measurement
}

\author{
Emma Wilson · Emiliano Rustighi · Philip L. Newland · Brian R. Mace.
}

\begin{abstract}
The isometric force response of the locust hind leg extensor tibia muscle to stimulation of a slow extensor tibia motor neuron is experimentally investigated, and a mathematical model describing the response presented. The measured force response was modelled by considering the ability of an existing model, developed to describe the response to stimulation of a fast extensor tibia motor neuron, to also model the response to slow motor neuron stimulation. It is found that despite large differences in the force response to slow and fast motor neuron stimulation, which could be accounted for by the differing physiology of the fibres they innervate, the model is able to describe the response to both fast and slow motor neuron stimulation. Thus, the presented model provides a potentially generally applicable, robust, simple model to describe the isometric force response of a range of muscles.
\end{abstract}

Keywords Muscle model - Isometric force - motor neuron · grasshopper

\section{Emma Wilson}

Institute of Sound and Vibration Research

University of Southampton

Southampton, Hampshire SO17 1BJ

United Kingdom

E-mail: emma.d.wilson@gmail.com

Emiliano Rustighi

Institute of Sound and Vibration Research

University of Southampton

Philip L. Newland

Centre for Biological Sciences

University of Southampton

Brian R. Mace

Department of Mechanical Engineering

University of Auckland

Institute of Sound and Vibration Research

University of Southampton

\section{Introduction}

Mathematical models of muscle contractile forces are often developed for a specific role or purpose so that their level of complexity is dependent on their application or the motivation for the model (Winters, 1995; Alexander, 2003). An overly specialised model, however, does not shed any light on general muscle mechanics since it is effectively an overfit that describes a very specific and well defined system. A muscle model capable of describing the response to different neural inputs provides a potentially generally applicable, robust strategy for modelling the muscle force response of a range of muscles across a range of species. Such a model could increase our understanding of the important processes involved in contraction. This study aims to test the robustness of a previously developed model by evaluating whether it is capable of describing the very different force profiles measured in response to stimulation of different motor neurons that both innervate the locust hind leg extensor tibia muscle (ETi).

The interaction between neural and muscular systems in the control of simple tasks, such as locomotion, is still poorly understood (Dickinson et al., 2000). By studying muscular function in a system where the neural components are simple and well understood, a greater insight into the neuromechanical system can be gained (Guschlbauer et al., 2007). Previous research into insect muscle has shown that the structure of the excitation contraction systems, and muscle are similar to those of any other striated muscle (Klowden, 2002). Insect muscle can, therefore, be used to gain insight into the basic properties of muscle. In this study the force response of the locust hind leg extensor tibia muscle (ETi) during stimulation of a slow extensor tibia motor neuron (SETi) is considered, and this response compared to the previously studied response to fast extensor tibia motor neu- 
ron (FETi) stimulation. These neurons independently excite the slow and fast fibers, contained within the same extensor tibia muscle. The neural networks that control this muscle have already been well described in the literature (Hoyle, 1955a,b, 1978; Burrows, 1996; Bennet-Clark, 1975; Burrows and Horridge, 1974; Newland and Kondoh, 1997). Furthermore, the slow axon can be stimulated independently of the fast axon as it leaves the ganglion by a different nerve (N.3b. as opposed to N.5.) (Usherwood, 1975). In most insect muscle the slow axons have higher thresholds for electrical stimulation and travel in the same nerve trunk as the fast axons, meaning the slow behaviour can only be studied during spontaneous or reflex actions, or by chance (Usherwood, 1975). Thus the locust ETi lends itself to studying fast and slow behaviours. The slow fibers are responsible for the majority of movements, with the function of the fast fibers being for jumping, kicking, or hopping (Heitler, 1977; Burrows, 1996; Hoyle, 1978).

The slow and fast motor neuron responses of muscles have been found to be different (Baylor and Hollingworth, 2003; Klowden, 2002; Hoyle, 1978). The response to slow motor neuron activation is characterised by very weak twitch contractions that generate only a small proportion of the maximum force at high stimulation rates. The responses to multiple contractions sum to give contractions that are much stronger at high stimulation frequencies than at low frequencies, relaxation rates are relatively long, and the response is reasonably resistant to fatigue (Hoyle, 1978). In contrast the response to fast axon stimulation fatigues relatively quickly, twitch responses are brisk and strong with the tetanus:twitch force ratio being of the order of about 10:1 (Hoyle, 1978). These differences can likely be attributed in part to fast muscle fibers having shorter sarcomeres, more extensive Sarcoplasmic Retictulum (SR) and a 3:1 ratio of actin:myosin filaments (Klowden, 2002). Slow muscle fibers have less SR, longer sarcomeres and a 6:1 ratio of actin:myosin (Klowden, 2002).

A model has been previously developed to describe the isometric force response of ETi to fast extensor tibia motor neuron (FETi) activation (Wilson, 2010; Wilson et al., 2011). In this study the isometric force response to SETi stimulation is analysed, and the ability of the model, developed to describe FETi stimulation, to also describe the response to SETi stimulation considered. The responses to each stimulation type are expected to generate very different behaviours, with the twitch to tetanus ratios expected to range from almost zero in the response to SETi stimulation up to approximately 0.15 in the response to FETi stimulation (Usherwood, 1975). Although different behaviours will again be seen in other species, testing the model's ability to describe such different responses provides a good test of its robustness and ability to fit to different types of data. This is likely to be transferable to other species, especially given that the differences between the response of the locust ETi muscle to FETi and SETi stimulation may be greater than the differences across some species. For example the twitch to tetanus ratio and the frequency at which maximum force is reached in response to the FETi stimulation are of the order of 0.15 and $70 \mathrm{~Hz}$ respectively (Wilson et al., 2011): these values are similar to the values of $0.1-0.7$ and $20-60 \mathrm{~Hz}$ (Enoka, 2008) measured in human muscles, but very different to the SETi response in which no saturation has occurred at $70 \mathrm{~Hz}$, and twitch to tetanus ratios are negligible.

\section{Materials and Methods}

Adult locusts (Schistocerca gregaria, Forskål) of both sexes, taken from a colony at the University of Southampton, were used for experiments.

\subsection{Preparation for SETi measurements}

Locusts were securely fixed, ventral side up in modelling clay. A small window was cut in the ventral thorax cuticle and underlying airsacs removed to expose the nerves supplying the left (ventral view with head at top) metathoracic leg. A pair of small hook electrodes were placed under nerve $N .3 b$. in the thorax and insulated with petroleum jelly. The remainder of the open thorax was bathed in saline. These hook electrodes were used to excite SETi by delivering stimulation voltage pulses of $3 \mathrm{~ms}$ duration. The electrodes could also be used to record the nerve activity.

The extensor muscle apodeme was exposed for length measurements by cutting a small window at the distal end of the femur, close to the femoral tibial (FT) joint. The flexor muscle apodeme and airsacs were removed to expose the ETi apodeme. The ETi apodeme was clamped in the forceps, close to the FT joint. The apodeme distal to the forceps was cut and the tibia removed. The forceps were mounted so that the force they measured was in line with the global direction of muscle force (i.e. in line with the apodeme). Prior to measurement, the ETi muscle was extended until it was just in tension when the input to the muscle was zero, i.e. the muscle set to its resting length.

\subsection{Recording evoked SETi force response}

Six locusts with mean length of $48.0 \pm 1.2 \mathrm{~mm}$ and mass of $2.05 \pm 0.14 \mathrm{~g}$ were used to measure the force response to direct SETi stimulation. To stimulate SETi, the amplitude of 
the voltage pulses was set just supra threshold to elicit a twitch when a spike in SETi was evoked. The resultant muscle tension was measured by clamping the extensor muscle apodeme in a pair of forceps attached to a S100 SMD thin film load cell with range up to $20 \mathrm{~N}$ (Strain Measurement Devices, USA). The load cell forceps system deforms linearly with force, at the maximum recorded tension $(0.5 \mathrm{~N})$ the deflection of the system was measured to be $0.1 \mathrm{~mm}$; considering that the total range of movement of this muscle is of the order of $\pm 0.5 \mathrm{~mm}$ there is likely to be some departure from the assumption of isometric conditions when measuring larger forces. The consequence of this is that there are likely to be small errors in the measurement of the force when the forces being measured are high.

The output of the load cell was fed into a Flyde FE-396TA transducer amplifier to low-pass filter (cut-off frequency $1 \mathrm{KHz}(-3 \mathrm{~dB}))$ and amplify the signal prior to acquisition. The force output (and corresponding input stimulus signal) was recorded using Spike2 software at a sampling frequency of $10,417 \mathrm{~Hz}$ on a Cambridge Electronic Design $1401 \mathrm{~A} / \mathrm{D}$ converter. This sampling frequency is much higher than necessary for the force signal. However, it was chosen to be consistent with the frequency at which the neural signal was sampled. The actual muscle force was calculated by calibrating the forceps load-cell system using weights hung from the forceps such that the force due to the weights had the same line of action relative to the forceps load cell system as did the muscle force. This was carried out for a range of loading and unloading conditions, and voltage output plotted against the force. A straight line was fitted and a calibration factor obtained to convert voltage to force.

\subsubsection{Muscle inputs}

Activity of the SETi motor neuron is associated with slow behaviours such as walking and scratching (Hoyle, 1955b). Recordings of SETi activity during a scratch made by Zakotnik et al. (2006) were used to provide physiologically relevant inputs and to determine general properties of the SETi activity, namely minimum, maximum and average spike frequencies. The maximum and minimum instantaneous spike frequencies across $3 \mathrm{~s}$ of recording during a scratching movement were measured as $500 \mathrm{~Hz}$ and $10 \mathrm{~Hz}$ respectively, with the average spike frequency being $50 \pm 10 \mathrm{~Hz}$.

The muscle was stimulated with single isolated stimuli, constant frequency pulse trains (CFTs), general nonconstant frequency pulse trains (NCFTs) and physiologically relevant NCFTs. Twenty pulse CFTs with interpulse frequencies (IPFs) of 10, 13, 20, 40, and 67Hz were used. General NCFTs consisted of a 29 pulse train which increased and then decreased in frequency (to the initial value), with a maximum frequency of $100 \mathrm{~Hz}$ and minimum of $10 \mathrm{~Hz}$, and a 60 pulse train which decreased and then increased in frequency (again to the initial value) with a minimum frequency of $1 \mathrm{~Hz}$ and maximum of $50 \mathrm{~Hz}$. The physiologically relevant NCFT inputs were taken directly from Zakotnik et al. (2006).

This stimulus range was chosen as it contains a rich range of frequencies that coincide with the frequency of SETi activity, this enables the model to be tested across a range of relevant inputs. No frequencies as high as the maximum measured instantaneous frequency of $500 \mathrm{~Hz}$ (as measured from Zakotnik et al. (2006)) were used in the CFTs or general NCFTs as on average the frequency of SETi stimulation is a lot lower (from Zakotnik et al. (2006)). The data was split into training and test data. Training data consisted of a twitch, a 20 pulse $67 \mathrm{~Hz}$ CFT and the two general NCFT pulse trains (described above) and was used to estimate the model parameters. The particular training data was chosen as it stimulates a rich range of behaviours, whilst keeping the size of the training data small. The test data can be split into general and physiologically relevant data and was used to ensure that the model does not overfit the data and to assess the ability of the model to predict the force response to different inputs. The SETi response was trained separately to the FETi response, to estimate two different sets of model parameters to describe each behaviour.

\subsection{Recording voluntary SETi force response}

A separate set of four locusts were used to measure the force response to voluntary SETi inputs. The locusts were prepared as described above, and the end of a paintbrush used to brush the locusts head to stimulate voluntary SETi activity. The hook electrodes under nerve $N .3 b$. were set to record the activity of the nerve and this output was amplified and recorded using Spike2 software with a sampling frequency of $10,417 \mathrm{~Hz}$ on a Cambridge Electronic Design 1401 A/D converter. The corresponding muscle force was measured by the load-cell forceps system described above.

\subsection{Recording evoked FETi force response}

The method of measuring the response to FETi stimulation, to provide a comparison to the SETi response, was reported in detail in (Wilson et al., 2010; Wilson, 2010). Briefly, a separate set of five adult locusts to those used for SETi measurement, with mean length of $46.4 \pm 2.3 \mathrm{~mm}$ and mass of $1.73 \pm 0.30 \mathrm{~g}$, were taken from the colony at Southampton University. The locusts were again fixed ventral side up in modelling clay. The left (ventral view with head at top) hind 
leg was clamped ventral side up with the femur securely fixed and the tibia free to move. The angle between the femur and the tibia was set to $80^{\circ}$ with a pin. The extensor muscle was stimulated directly with implanted electrodes with pulses of $3 \mathrm{~ms}$ duration and approximately $5 \mathrm{~V}$ magnitude. The method of stimulation differs to the direct nerve stimulation used to excite SETi, this is due to the fact that FETi can be stimulated at a lower threshold than SETi. An S100 SMD thin film load cell with range up to $1 \mathrm{~N}$ was used to measure the force at the tibia. The output of the load cell was fed into a Flyde FE-396-TA transducer amplifier to amplify and low-pass filter the signal prior to acquisition, with a cut-off frequency of $1 \mathrm{KHz}(-3 \mathrm{~dB})$, and a sampling frequency of $2.2 \mathrm{KHz}$.

The method of measuring the force produced by the ETi in response to FETi and SETi stimulation differed due to the different force magnitudes associated with each motor neuron type. When the force response to a single SETi stimulus pulse was measured at the tibia the resultant twitch force was too small to resolve (see also Hoyle (1978)). On the other hand, the large muscle force generated in response to FETi stimulation meant that for large inputs the deflection of the load cell, and the departure from isometric conditions, was significant when the force was measured at the apodeme in the same way as described for SETi responses. Furthermore, for high forces the apodeme often snapped at the point of attachment to the forceps.

\subsection{Force Model}

A previous comparison of the ability of seven different models to describe the isometric force response of the locust hind leg ETi muscle to FETi stimulation found an 'Adapted model' developed by the authors to perform best (with the measure of best quantified by the least squares error between model and measurement and Aikaike's and Bayesian Information Criteria) (Wilson et al., 2011). A simplified version of this model (Simplified Adapted model) also provided reasonable fits to data with one less model parameter. The ability of these two models to also describe the isometric response to SETi stimulation is considered. These models give the muscle force $F(t)$ as an output and take an input pulse train $u(t)$ as an input where,

$u(t)=\sum_{i=1}^{n} \delta\left(t-t_{i}\right)$

where $n$ defines the number of input pulses, $t$ the time and $t_{i}$ the time at which the $i^{t h}$ pulse occurs. Using this as an input makes the assumption that the input pulses can be approximated as impulses. The Adapted model equations are

$$
\begin{array}{r}
\dot{C}_{N}(t)+\frac{C_{N}(t)}{\tau_{c}}=u(t) \\
x(t)=\frac{C_{N}(t)^{m}}{C_{N}(t)^{m}+k^{m}} \\
\dot{F}(t)+\frac{F(t)}{\tau_{1}+\tau_{2} x(t)}=A x(t)
\end{array}
$$

This model has 6 parameters, $\tau_{c}, \tau_{1}, \tau_{2}, m, k, A$ and consists of a first order linear ordinary differential equation, followed by a differential equation that includes non-linear saturation terms. The variable $x(t)$ is an intermediate stage in the model and describes the non-linear saturation, with the parameters $m$ and $k$ defining the shape of this non-linearity. The variable $C_{N}$ is another intermediate stage in the model that is likely to be a measure of the free calcium concentration. The parameters $\tau_{c}, \tau_{1}$ and $\tau_{2}$ are time constants, and $A$ a gain. The Adapted model was developed from the model of Ding et al. (2002) and has a similar form to the model of Bobet and Stein (1998). The development of the model is discussed in more detail in Wilson et al. (2011); Wilson (2010). In the Simplified Adapted model $\tau_{2}$ is assumed to be zero, and so the model equations become

$$
\begin{gathered}
\dot{C}_{N}(t)+\frac{C_{N}(t)}{\tau_{c}}=u(t) \\
x(t)=\frac{C_{N}(t)^{m}}{C_{N}(t)^{m}+k^{m}} \\
\dot{F}(t)+\frac{F(t)}{\tau_{1}}=A x(t)
\end{gathered}
$$

\subsubsection{Estimation Algorithm}

Parameters were estimated by finding the values that gave the smallest least squares error between the model output and measured ETi force data, when using the complete set of training data. The parameters for each locust were optimised separately due to the high variability of the force data. This was done using the MATLAB function lsqnonlin and a fixed step ordinary differential equation (ODE) solver. The step size of the solver was equal to the sampling time of the data, i.e. $9.6 \times 10^{-5}$. Initial conditions were set to zero and a fourth order Runge-Kutta approximation was used. An initial estimate of parameter values was made and an iterative method using a trust-region-reflective algorithm was then used to find the parameters which provide the best fit to data (The MathWorks Inc, 2001). The parameter optimisation routine was re-run using a range of initial parameter estimates to avoid estimating local minima. Initial parameters were randomly varied about fixed points, this was done by generating initial estimates from normal distributions with given standard deviations and mean values for each parameter. The standard deviation and means used to generate initial estimates of each parameter are summarised in Table 1. 
For each locust the estimation routine was rerun with seven different start values. Functions were evaluated until lower bounds on the step size or change in object value during a step were met. These bounds were defined by setting the lower bound on step size (TolX) and the lower bound on change in object value (TolFun) to $1 \times 10^{-7}$ (tolerances are an order of magnitude smaller than those used previously in modelling the response to FETi stimulation (Wilson et al., 2011). Tighter tolerances were used to ensure that the final estimates converged for the full range of intial parameter values). If the maximum allowed number of function evaluations was exceeded before these limits were reached the optimisation was re-started from the final values until the specified tolerances were reached.

Table 1 Mean and standard deviation of the normal distribution used to randomly generate each initial parameter.

\begin{tabular}{l|cccccc} 
& $\tau_{1}$ & $\tau_{1}$ & $\tau_{2}$ & $k$ & $A$ & $m$ \\
\hline Mean & 0.1 & 0.1 & 0.1 & 5 & 10 & 2 \\
Standard deviation & 0.1 & 0.1 & 0.1 & 1 & 1 & 0.5
\end{tabular}

Presented errors, $E$, between the data and estimated model are calculated as,

$E=\frac{\sum_{i}\left(F_{i}-\hat{F}_{i}\right)^{2}}{\sum_{i}\left(F_{i}\right)^{2}}$

where $\hat{F}_{i}$ is the model estimate and $F_{i}$ the actual data at the $i$ th time step, the sums being taken over all measured data points. The errors minimised during the fitting routine are defined as $\sum_{i}\left(F_{i}-\hat{F}_{i}\right)^{2}$. As the length of training data is fixed this is equivalent to minimising the error as defined in Eq. (8). In Sec. 3 the data is split into all data, training data, general test data and physiological type data and the errors are given for each group of data.

\section{Results}

\subsection{Force Measurements}

The force response of the ETi muscle and corresponding neural activity in nerve N.3b. during 'voluntary movements' (i.e. no external stimulation) were recorded (Fig. 1). Recordings of SETi activity in nerve N.3b. (Fig. 1) reveal that SETi can be spontaneously active with each spike followed by a small contraction of the ETi muscle lasting from 0.3-0.5s and with an amplitude of $0.005-0.01 \mathrm{~N}$. Where spikes are closely spaced (less than $0.5 \mathrm{~s}$ apart) the force responses sum non-linearly with the maximum force reaching around $0.03 \mathrm{~N}$
(Fig. 1). A slight delay of 0.013 s exists between the neural and force recordings. This lag between muscle force production and muscle activation is well known to exist, and is termed electromechanical delay (EMD) (Cavanagh and Komi, 1979). The force responses were time shifted by $0.013 \mathrm{~s}$ to remove the EMD between input and output signals for further analysis.

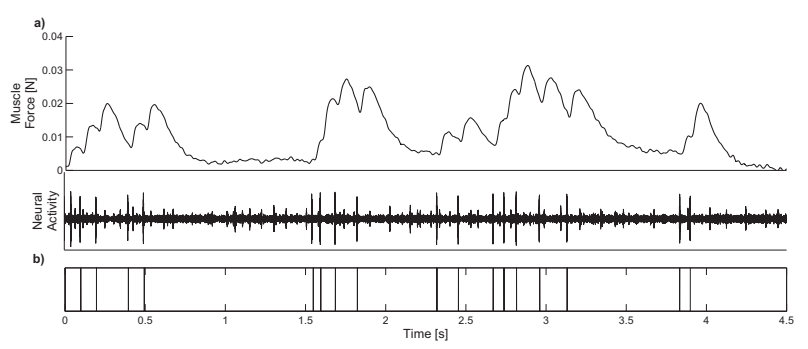

Fig. 1 a) Example of neural activity recorded from nerve $N .3 b$. in the thorax and the corresponding muscle force, b) simplification of neural recording, used as model input, each pulse above a threshold that causes an increase in muscle force is approximated as a single pulse of $3 \mathrm{~ms}$ duration.

\subsubsection{Twitch Response}

The evoked twitch response to SETi stimulation is shown for one locust in Figure 2a and a further six locusts in Figure $2 \mathrm{c}$. The total duration of the contraction ranges between $0.3-0.5 \mathrm{~s}$ with the peak force ranging from $0.003-0.013 \mathrm{~N}$ and occurring after approximately $0.06 \mathrm{~s}$. These measurements are consistent with responses measured during spontaneous activation of SETi (Fig. 1). The following measurements (as defined by Frey Law and Shields (2005)) were used to quantitatively characterise each twitch response: peak force (PF), defined as the maximum recorded force; force-time integral (FTI), defined as the area under the force-time trace; half relaxation time (HRT), defined as the time for the force to decay from $90 \%$ to $50 \%$ of the peak force; late relaxation time (LRT), defined as the time for the force to decay from $40 \%$ to $10 \%$ of the peak force; and the time to peak tension (TPT) defined as the time required to reach $90 \%$ of the peak force from time zero. These measurements are given in Table 2. On average the SETi twitch response has a peak force of $0.007 \mathrm{~N}$ and takes $53 \mathrm{~ms}$ to reach $90 \%$ of the maximum tension, with relaxation taking over twice as long. The measurements show variation of up to $500 \%$ between locusts.

The twitch response to SETi stimulation can be compared to the response to FETi stimulation (Fig. 2b, 2d, and Table 2). The peak force recorded in response to SETi stimulation is of the order of 100 times smaller than that recorded in response to FETi stimulation, with the contraction times being on average 1.5 times greater for the SETi response. 

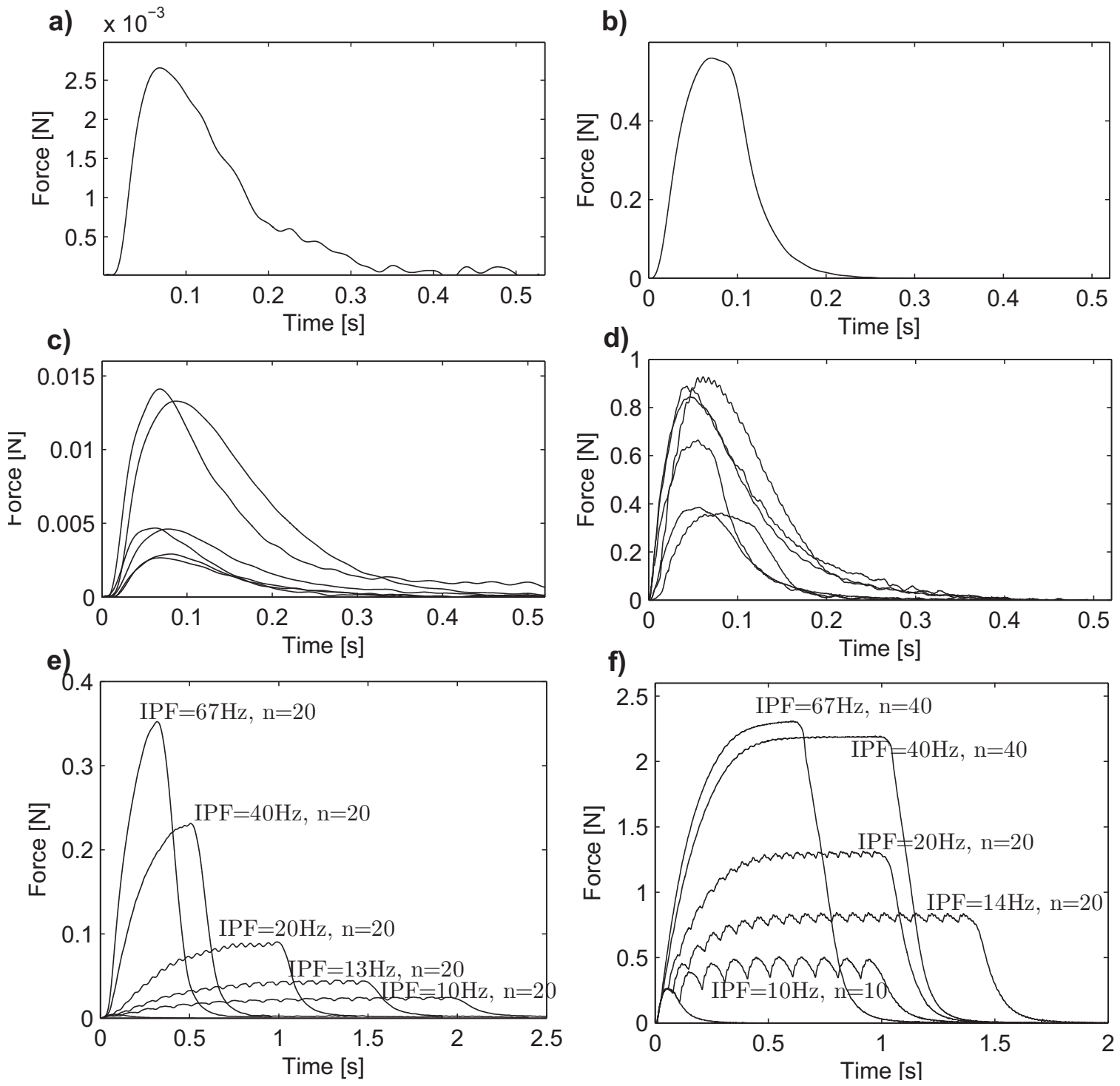

Fig. 2 Comparison between the force response of ETi to SETi and FETi stimulation, the peak twitch force measured in response to SETi stimulation is approximately 100 times less than that measured in response to FETi stimulation (note widely different scales between SETi and FETi are used for the force axes). a) Twitch response to SETi stimulation, b) twitch response to FETi stimulation in the same locust as a), c) twitch response to SETi stimulation of six different locusts, d) twitch response to FETi stimulation in a different set of six locusts. e) response of one locust to constant frequency (CFT) stimulation of SETi for inputs with different interpulse frequencies (IPF) and numbers of input pulses (n), f) response of one locust to CFT stimulation of FETi for inputs with different interpulse frequencies (IPF) and numbers of input pulses (n).

\subsubsection{Response to Constant Frequency Pulse Trains}

The response to CFT stimulation of SETi for a range of pulse frequencies (i.e. a range of different interpulse intervals (IPIs)) for one locust is shown in Figure 2e, with the response of six different locusts shown in Figure 3. Variability exists between locusts. However, the responses are qualitatively similar. The twitch force is much smaller than the summed forces, with the peak twitch force being approximately 100 times smaller than the peak force in response to a 20 pulse CFT with $67 \mathrm{~Hz}$ IPF. The maximum force in- creases with frequency, no maximum force appears to have been reached when the IPF is $67 \mathrm{~Hz}$. For a comparison, the response to CFT stimulation of FETi for one locust is provided in Figure 2f. For all frequencies a constant force level is reached faster in response to FETi stimulation, the SETi responses sum to increase the force level over a greater number of input pulses (for the higher frequency CFTs fewer pulses are applied during SETi stimulation. However, after an equivalent number of pulses the FETi response still appears closer to reaching a constant force level). In the FETi response there is less of a difference between the twitch 
Table 2 A comparison of the properties of the ETi twitch response to SETi and FETi stimulation. The measured twitch characteristics are peak force (PF), force time integral (FTI), half relaxation time (HRT), late relaxation time (LRT), and time to peak tension (TPT) and defined in the text.

\begin{tabular}{|l|c|c|c|c|c|}
\hline Locust & PF (N) & FTI (Nms) & HRT (ms) & LRT (ms) & TPT (ms) \\
\hline FETi & & & & & \\
LFa & 0.84 & 99.4 & 45.5 & 95.2 & 31.3 \\
LFb & 0.39 & 38.8 & 30.4 & 62.6 & 35.0 \\
LFc & 0.66 & 56.9 & 20.2 & 52.0 & 35.0 \\
LFd & 0.89 & 108.4 & 52.4 & 114.1 & 31.7 \\
LFe & 0.93 & 114.7 & 51.5 & 62.6 & 46.0 \\
LFf & 0.36 & 40.7 & 32.7 & 30.8 & 50.6 \\
\hline SETi & & & & & \\
LSa & 0.013 & 2.3 & 71.3 & 112.4 & 61.6 \\
LSb & 0.003 & 0.4 & 49.7 & 87.4 & 58.6 \\
LSc & 0.003 & 0.4 & 62.9 & 122.7 & 51.4 \\
LSd & 0.005 & 0.7 & 60.0 & 155.9 & 57.1 \\
LSe & 0.005 & 0.6 & 49.0 & 97.9 & 40.0 \\
LSf & 0.014 & 2.1 & 56.0 & 135.7 & 50.8 \\
\hline Average & & & & & \\
Mean FETi & 0.68 & 76.5 & 38.8 & 69.6 & 38.3 \\
Mean SETi & 0.007 & 1.1 & 58.2 & 118.7 & 53.3 \\
\hline
\end{tabular}

magnitude and maximum tetanic force, with the peak twitch force being approximately 10 times smaller than the peak force in response to a 20 pulse CFT with $67 \mathrm{~Hz}$ IPF. Saturation of the maximum force level is close to occurring in the FETi response, in that there is almost no further increase in the maximum force level when the stimulation frequency is increased from $40 \mathrm{~Hz}$ to $67 \mathrm{~Hz}$. This saturation is not evident in the SETi responses, in all cases there is a significant increase in the force level with input frequency.

\subsubsection{Response to Non-Constant Frequency Pulse Trains}

The response to NCFT stimulation of SETi for a range of input types and six different locusts is shown in Figure 4. Figures $4 \mathrm{a}$ and $\mathrm{b}$ show the responses to general NCFT inputs and Figures $4 \mathrm{c}-\mathrm{f}$ the response to physiologically relevant SETi type inputs. Again, the specific details of the responses vary from one locust to another, yet the responses are qualitatively similar. The durations of responses to the SETi type inputs are of the order of $1 \mathrm{~s}$, with the maximum recorded force of the order of $0.4 \mathrm{~N}$. During walking movements it is SETi that is active, step periods are commonly 200-500ms (Usherwood and Runion, 1970), the time courses of the responses given in Figures $4 c-f$ are of the same order of magnitude. The SETi signals were taken from scratching recordings made by Zakotnik et al. (2006) who measure resultant movements that last for around 1s, so the time courses of contraction presented in Figure $4 \mathrm{c}-\mathrm{f}$ seem reasonable. The measured force levels are consistent with the observation that the maximum forces measured in response to SETi stimulation are of the order of $1 \mathrm{~N}$ (Hoyle, 1955b).

\subsection{Modelled Force}

The estimated parameters that give the lowest errors between the estimated and actual SETi responses across the training data for five of the six locusts are presented in Table 3 for both the Adapted and Simplified Adapted models. Due to voluntary movements affecting the force response whilst recording training data in one of the six locusts (Lsb), a complete set of training data could not be recorded, and consequently no model parameters were estimated for this locust. For the range of starting parameters, the final values of the parameters were within $0.01 \%$ of each other, with small variations due to the tolerances on the convergence conditions. This suggests that global, as opposed to local minima are estimated. Furthermore, once estimated, each parameter was manually varied in turn and the resulting change in error plotted against the change in parameter value. All of the variations of estimated error as functions of the parameters had slopes which were monotonic with single minima, which also indicates that global minima are estimated. The errors given in Table 3 are calculated as described by Eq (8). The errors refer to the error in fit to all data $\left(E_{\text {all }}\right)$, error in fit to training data $\left(E_{\text {train }}\right)$, error in fit to all data except the training data $\left(E_{\text {test }}\right)$ and the error in fit to the physiologically relevant data $\left(E_{\text {seti }}\right)$. The average parameters estimated when fitting to the FETi response in a separate set of six locusts (Wilson et al., 2011) and the relevant errors are also provided in Table 3. For each locust all errors ( $E_{\text {all }}, E_{\text {train }}$, $\left.E_{\text {test }}, E_{\text {seti }}\right)$ are of comparable magnitudes, so that the estimates are not particularly sensitive to the set of data used for the estimations. Furthermore, sometimes the errors for 

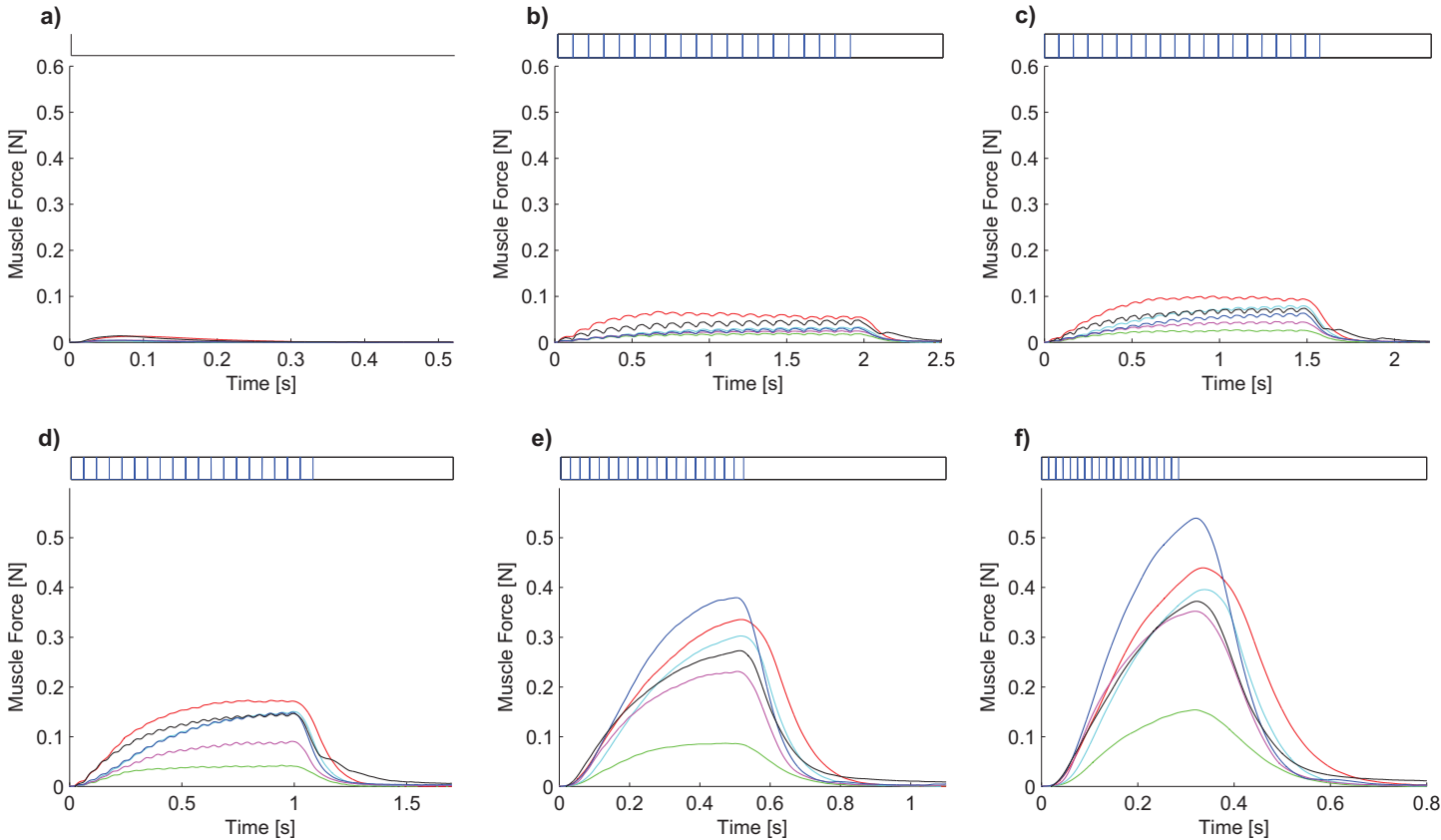

Fig. 3 Muscle force recorded in six different locusts (each trace represents a different locust) in response to constant frequency SETi stimulation with the input pulse trains shown above each force response a) Twitch response, b) response to 20 pulse CFT with IPI=0.1s (IPF=10Hz), c) response to 20 pulse CFT with $\mathrm{IPI}=0.075 \mathrm{~s}(\mathrm{IPF}=13 \mathrm{~Hz}$ ), d) response to 20 pulse CFT with $\mathrm{IPI}=0.05 \mathrm{~s}(\mathrm{IPF}=20 \mathrm{~Hz})$, e) response to $20 \mathrm{pulse} \mathrm{CFT}$ with $\mathrm{IPI}=0.025 \mathrm{~s}(\mathrm{IPF}=40 \mathrm{~Hz}), \mathrm{f})$ response to 20 pulse $\mathrm{CFT}$ with $\mathrm{IPI}=0.015 \mathrm{~s}(\mathrm{IPF}=67 \mathrm{~Hz})$.

the test and SETi data are lower than those for the training data used to estimate the parameter values. This indicates that the model is good and does not overfit to the training data.

There is some variability in the estimated parameters, consistent with variability in the force responses between animals. The parameter $A$, which is a constant gain, varies by around $700 \%$ for the Adapted model, and $400 \%$ for the Simplified Adapted model. However, this is reasonable considering the variation in PF seen in the twitch measurements is about $500 \%$ (Table 2). For the fit to SETi data using the Adapted model the parameter $\tau_{2}$ varies between being small and negative and small and positive, resulting in $\tau_{2}$ being on average 0.00. In most locusts, with the exception of Locust LSa, the Adapted model errors are only marginally better than the Simplified Adapted model errors, and greater differences are seen in the FETi fits. The main difference between the parameters estimated for the response to SETi and FETi stimulation is in the estimate of $k$ which is an order of magnitude larger when fitting to SETi data. Some of the estimates for the parameter $A$ estimated for responses to SETi stimulation are also much greater than the average value of $A$ for the fit to FETi data. The estimates for $\tau_{c}$ are consistently slightly larger in the SETi response, with the estimates of $\tau_{1}$ being consistently smaller. The parameter $m$ does not show much dependency on stimulation type. Differences between estimates of $m$ for the SETi and FETi response are small enough that they could be due to the fact that different sets of locusts were used to estimate the SETi and FETi parameters.

The errors in the fit to SETi data are consistently greater than those when fitting to FETi data, suggesting the models are better at describing the FETi response. However, the errors in fit to SETi data are still low, especially when considering the fit to physiologically relevant data where average estimated errors are $1.65 \%$ and $1.72 \%$ for the Adapted and Simplified Adapted models respectively. The increased error in fitting to SETi data could be due to the fact that the behaviour in response to SETi stimulation has a much greater range, with twitch forces being very small in comparison to cases where stimuli are closely spaced and sum. The errors and estimated parameters are discussed further in Sec. 4.

Figure 5 shows the fit to CFT inputs using both models in locust LSa. With the exception of the twitch response, the force response estimated by both models is very similar, the modelled force responses almost lie on top of each 

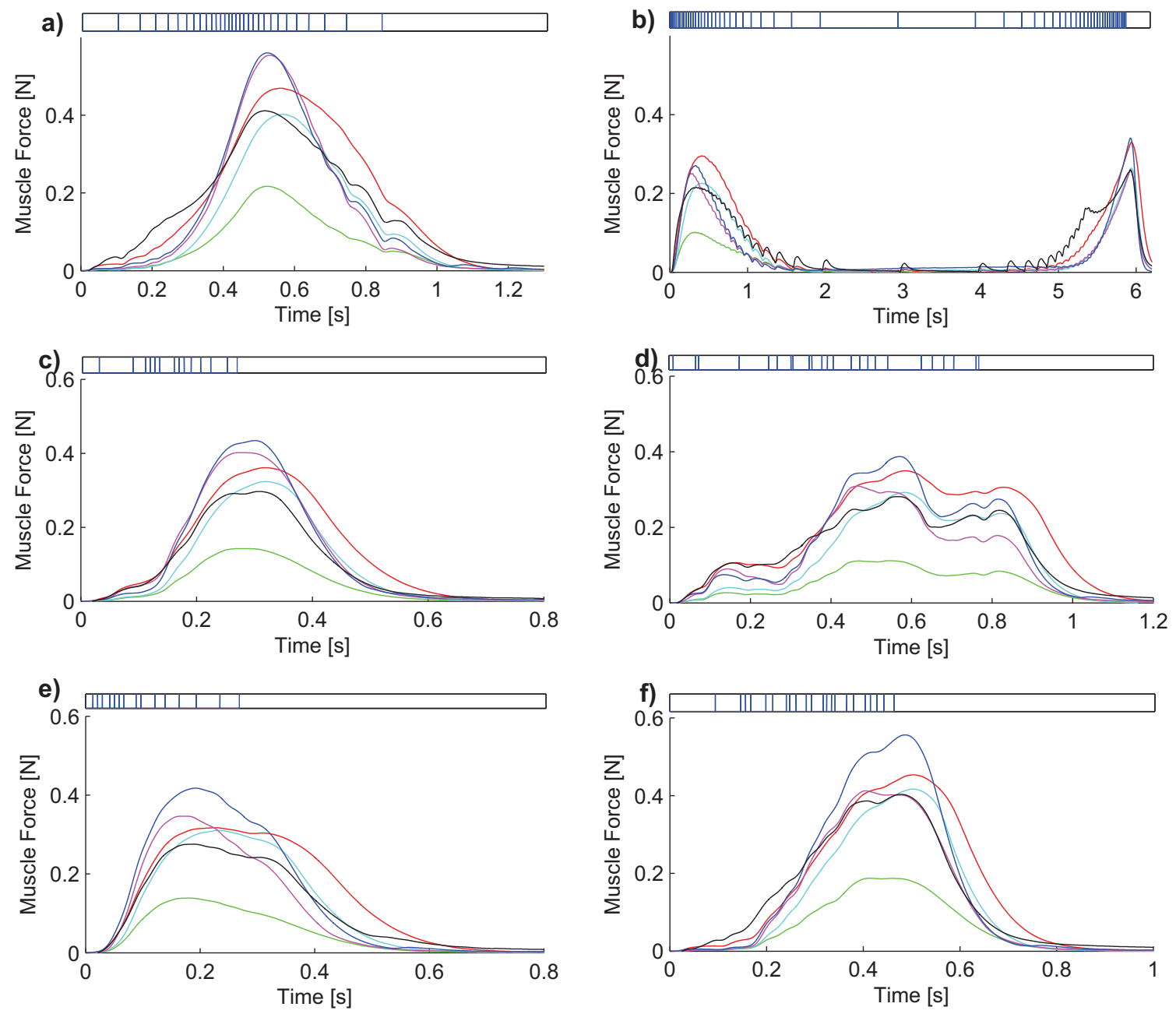

Fig. 4 Muscle force recorded in six different locusts (each trace represents a different locust) in response to NCFT SETi stimulation. The input pulse patterns are given above each force response. a,b) response to general non-constant frequency inputs, c-f) response to typical physiological SETi inputs, input pulses were taken from the SETi recordings shown in Figure 3 of Zakotnik et al. (2006).

other, hence differentiating the response to each model is hard. Both the Simplified Adapted and Adapted models provide a good description of the behaviour.

From Table 3 it is evident that the errors in fit are the lowest in the locust presented in Figure 5. In other locusts there were some discrepancies in the fit to low and mid frequency CFT inputs. Figure 6 is provided to show how the fit differs between locusts, and to demonstrate the problems with the fit in some locusts for intermediate frequency pulse trains. From Figure 6 it can be seen that in some locusts the models predict that the force rises too quickly and to the wrong force amplitude; this problem is evident for both the Adapted and Adapted Simplified models. In comparing the traces in Figure 6 it is evident that the responses that reach an apparent steady state are better described by the models. However, the response to SETi stimulation is expected to in- crease over a large number of pulses (Hooper et al., 2007a), as is the case in the force traces that are less well modelled, hence these traces may provide a better description of the expected behaviour. This suggests that the models are unable to capture the exact response to low or mid frequency pulse trains.

The fits to NCFT data using both the models for the locust with the lowest overall errors in fit (LSa) and that with the highest overall errors in fit (LSc) are given in Figure 7 and 8 respectively. In both cases the models provide a good description of the force response. Unlike for the mid frequency CFT trains, in which the model behaviour was different to the actual behaviour for LSc (Fig. 6b) the description of NCFT data is much better. Despite discrepancies in the fit to low-mid frequency CFT data, the fits to general NCFTs and physiologically relevant NCFTs are much better. 
Table 3 Estimated model parameters and associated errors for five locusts when fitting to SETi data using both the Adapted and Simplified Adapted models. The average FETi parameters and error for a different set of locusts is also shown for comparison.

\begin{tabular}{|c|c|c|c|c|c|c|c|c|c|c|}
\hline Locust & $\tau_{1}$ & $\tau_{1}$ & $\tau_{2}$ & $k$ & $A$ & $m$ & $E_{\text {all }}$ & $E_{\text {train }}$ & $E_{\text {test }}$ & $E_{\text {seti }}$ \\
\hline \multicolumn{11}{|c|}{ Adapted Model (SETi fit) } \\
\hline $\mathrm{LSa}$ & 0.10 & 0.07 & 0.06 & 3.21 & 5.53 & 1.76 & 1.08 & 1.06 & 1.09 & 0.98 \\
\hline $\mathrm{LSb}$ & - & - & - & - & - & - & - & - & - & - \\
\hline $\mathrm{LSc}$ & 0.12 & 0.04 & -0.01 & 10.90 & 42.64 & 1.90 & 2.82 & 2.57 & 3.03 & 1.93 \\
\hline LSd & 0.10 & 0.05 & -0.04 & 6.92 & 30.56 & 2.25 & 1.80 & 1.98 & 1.63 & 1.60 \\
\hline $\mathrm{LSe}$ & 0.10 & 0.05 & -0.04 & 6.92 & 30.54 & 2.25 & 2.38 & 1.62 & 2.97 & 2.01 \\
\hline LSf & 0.11 & 0.03 & 0.02 & 4.78 & 12.67 & 1.39 & 2.07 & 1.97 & 2.17 & 1.73 \\
\hline \multicolumn{11}{|c|}{ Simplified Adapted Model (SETi fit) } \\
\hline $\mathrm{LSa}$ & 0.12 & 0.08 & - & 4.32 & 8.19 & 1.89 & 1.20 & 1.11 & 1.27 & 1.25 \\
\hline $\mathrm{LSc}$ & 0.13 & 0.06 & - & 5.45 & 9.73 & 2.22 & 2.83 & 2.57 & 3.04 & 1.95 \\
\hline LSd & 0.12 & 0.04 & - & 9.53 & 34.65 & 1.90 & 1.82 & 1.99 & 1.66 & 1.62 \\
\hline LSe & 0.10 & 0.05 & - & 4.93 & 17.54 & 2.31 & 2.38 & 1.68 & 2.92 & 1.94 \\
\hline LSf & 0.12 & 0.04 & - & 6.52 & 18.06 & 1.44 & 2.09 & 1.98 & 2.20 & 1.86 \\
\hline \multicolumn{11}{|l|}{ Averages } \\
\hline SETi Adapted & 0.11 & 0.05 & 0.00 & 6.55 & 24.39 & 1.91 & 2.03 & 1.84 & 2.18 & 1.65 \\
\hline SETi Simplified Adapted & 0.12 & 0.05 & - & 6.15 & 17.63 & 1.95 & 2.06 & 1.87 & 2.22 & $\begin{array}{l}1.72 \\
E_{\text {kick }}\end{array}$ \\
\hline FETi Adapted & 0.070 & 0.083 & 0.10 & 0.57 & 5.8 & 1.8 & 1.05 & 1.30 & 0.80 & 0.82 \\
\hline FETi Simplified Adapted & 0.071 & 0.13 & - & 0.75 & 7.4 & 2.5 & 1.25 & 1.50 & 1.00 & 0.81 \\
\hline
\end{tabular}

As described in the Materials and Methods section, prior to stimulation the neural activity of nerve $N .3 b$. and the corresponding voluntary muscle force were recorded. This provides further physiologically relevant data to test the model's ability to describe the force response to stimulation of SETi. Figure 1a gives an example of the neural recordings and associated forces made in one locust. Large amplitude spikes in the neural recordings were approximated as impulses, and these used as model inputs. An example of the extracted model input is given in Figure 1b. Not every large amplitude spike in the SETi recording results in a contraction of ETi (for example the spike in the neural recording at $\mathrm{t}=0.04 \mathrm{~s}$ does not result in any contraction). The location of these spikes were not included in the input pulse trains.

Figure 9 shows the measured voluntary force and the resultant modelled force where the model input was extracted from the neural recording as shown in Figure 1. Both the Simplified Adapted, and Adapted models were used to simulate the responses. There is very little difference between the response predicted by each model, but the Simplified Adapted model has one fewer parameter. The sets of data shown in Figure 9a were used to train the model and estimate the model parameters, with the data in Figure $9 \mathrm{~b}$ used as test data. From Figure 9 it is clear that the Simplified Adapted model provides a good description of the response to voluntary SETi stimulation. The fit to training data is very good, and the model is also able to accurately predict the force response when applied to test data.

\section{Discussion}

\subsection{Variability of Responses}

Due to the variability of force responses a separate set of parameters is estimated for each locust. This variability between locust force measurements is also seen in other studies of insect muscle (Guschlbauer et al., 2007; Ahn and Full, 2002; Burns and Usherwood, 1979). This suggests that the variability is more likely to be a property of insect muscles, as opposed to an error in the preparation and force recording. Furthermore, the ETi muscle mass, total locust mass and dimensions of the locust were measured across a sample of six locusts taken from the colony. These values varied by up to $260 \%$ and so the variability in the force recordings can be explained, at least in part, by variability in the morphology of individual locusts. The correlation between the muscle mass (invasively measured) and locust mass (noninvasively measured) was found to be $0.42-0.98$ at the $95 \%$ confidence level. An extension to this work would be to establish if measurable variables such as locust mass are able to explain the force variations between locusts, and modify the model accordingly. However, a much larger set of locusts is required to study correlations to obtain small confidence intervals between measured model parameters, and measurable variables. 

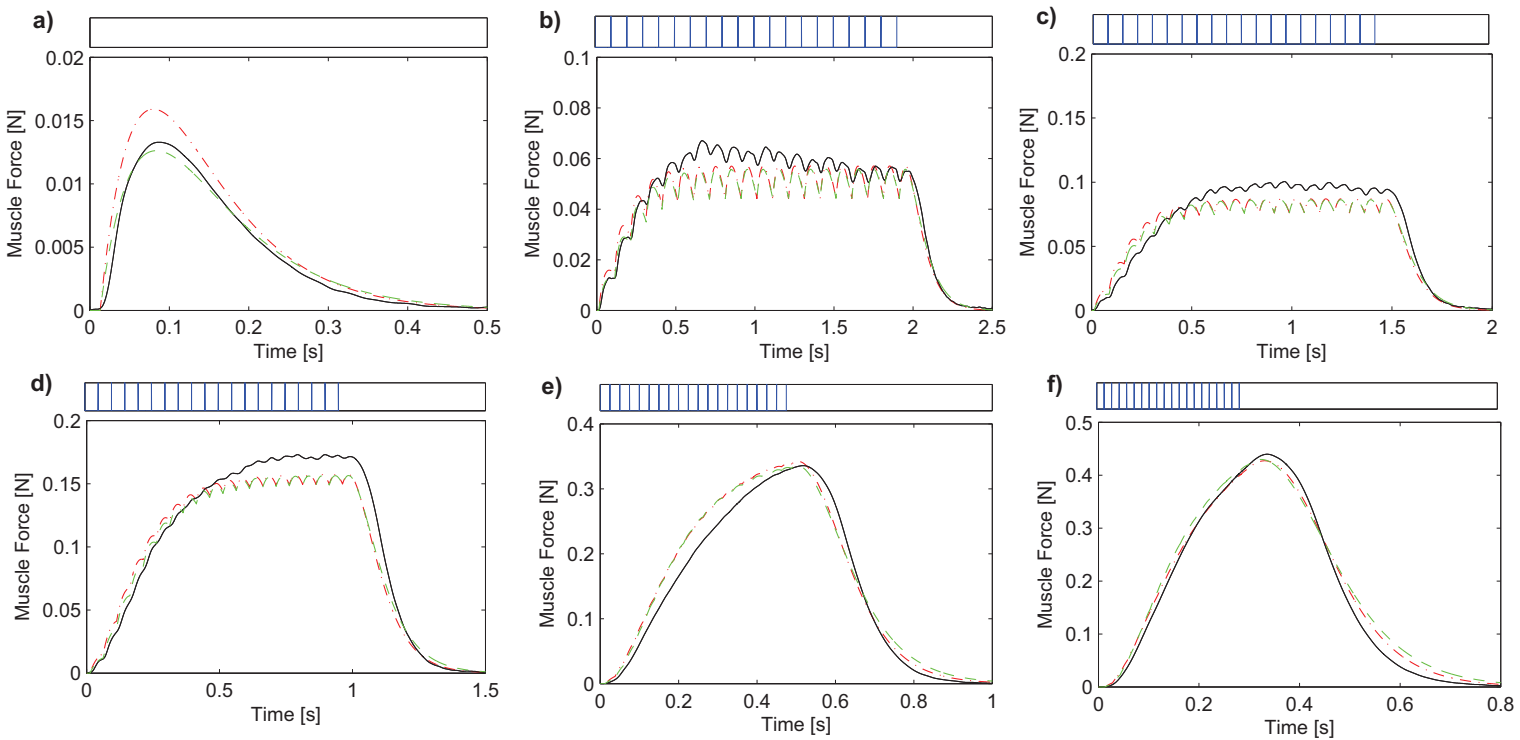

Fig. 5 Force response (-) and fit using the Adapted (-.-) and Simplified Adapted (- - ) models for locust LSa (the two modelled responses are very similar, hence, differentiating the two traces is hard). The corresponding input stimuli are shown above each force trace where the inputs were; a) single pulse, b) 20 pulse, $10 \mathrm{~Hz}$ CFT, c) 20 pulse, $13 \mathrm{~Hz}$ CFT, d) 20 pulse, $20 \mathrm{~Hz}$ CFT, e) 20 pulse, $40 \mathrm{~Hz}$ CFT, f) 20 pulse, $67 \mathrm{~Hz}$ CFT.

a)

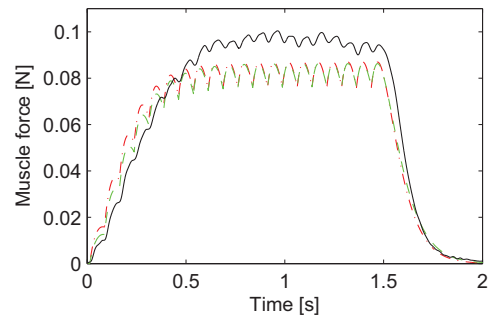

d)

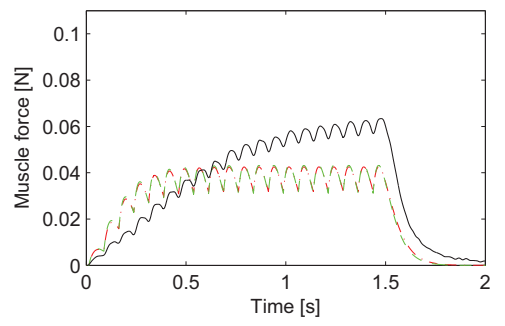

b)

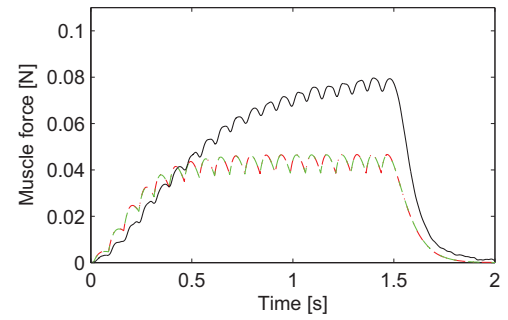

e)

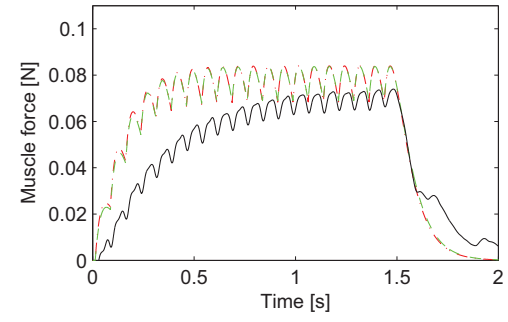

c)

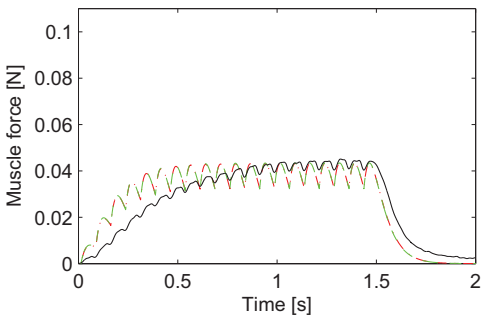

Fig. 6 Example of fit to the force response to a 20 pulse CFT with IPI=0.075s (-) across the ensemble of locusts using the Adapted (- . - ) and Simplified Adapted (- - ) models (the two modelled responses are very similar, hence, differentiating the two traces is hard). a) Locust LSa, b) Locust LSc, c) Locust LSd, d) Locust LSe, e) Locust LSf.

\subsection{SETi Response}

The SETi force response to a range of different inputs is presented above. Measured SETi forces range on average from a maximum of $0.007 \mathrm{~N}$ in response to a single stimulus up to around $0.4 \mathrm{~N}$ in response to a 20 pulse $67 \mathrm{~Hz}$ CFT. These measurements are consistent with previous studies that finds the twitch response to be just a few percent of the maximum force (Usherwood, 1975).

For high frequency SETi inputs the responses to individual pulses fuse together to produce smooth responses, however, the response to SETi stimulation, unlike that to FETi stimulation, doesn't appear to have saturated in maximum force level at the maximum input frequency of $67 \mathrm{~Hz}$. This is in contrast to mammalian muscles in which the responses to 

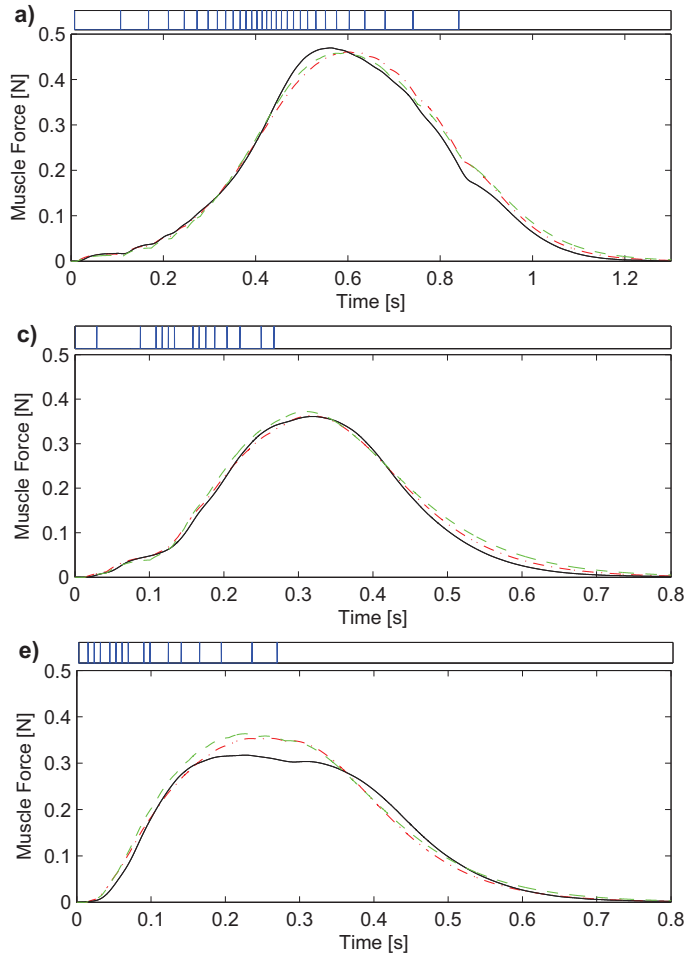
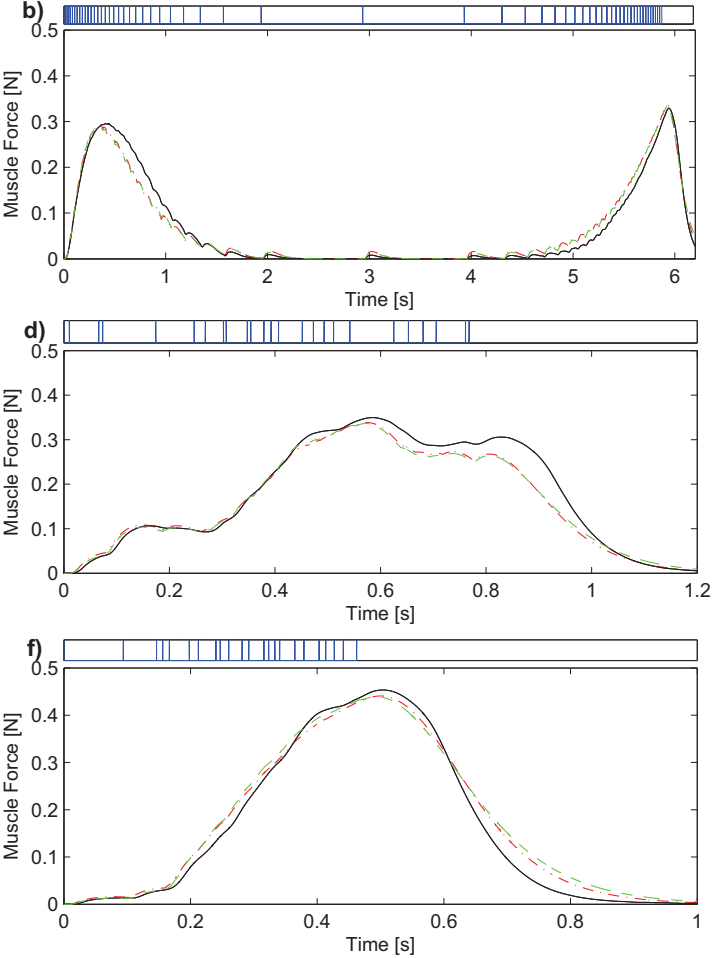

Fig. 7 Force response (-) and fit using the Adapted (-.-) and Simplified Adapted (- - ) models for locust LSa. The pulses occur at times indicated in the bar above each figure. a,b) The response to general NCFTs, c-f) response to physiological SETi type inputs.
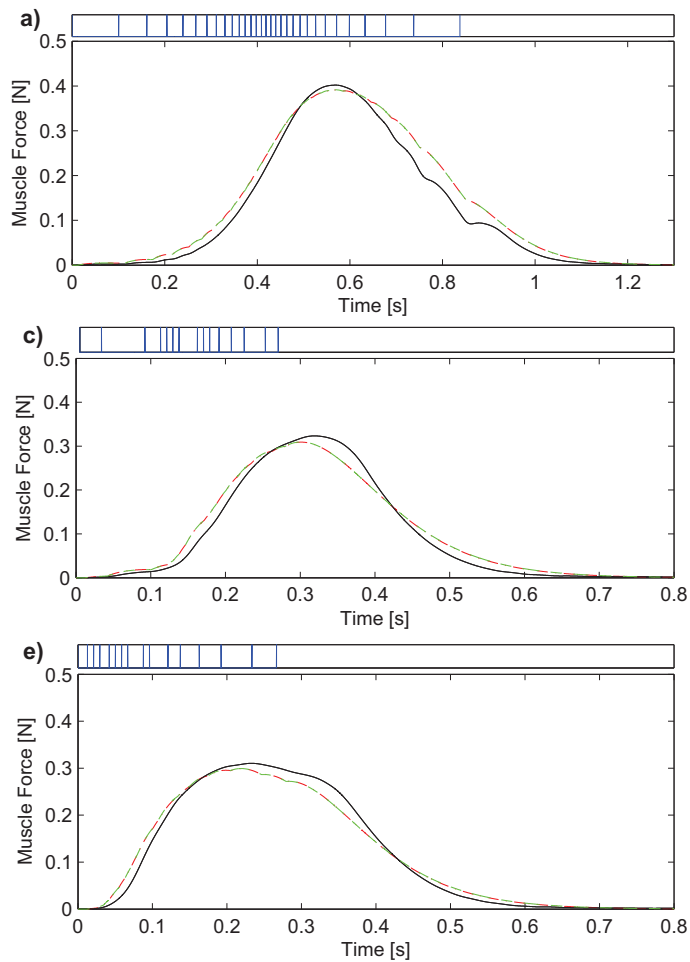
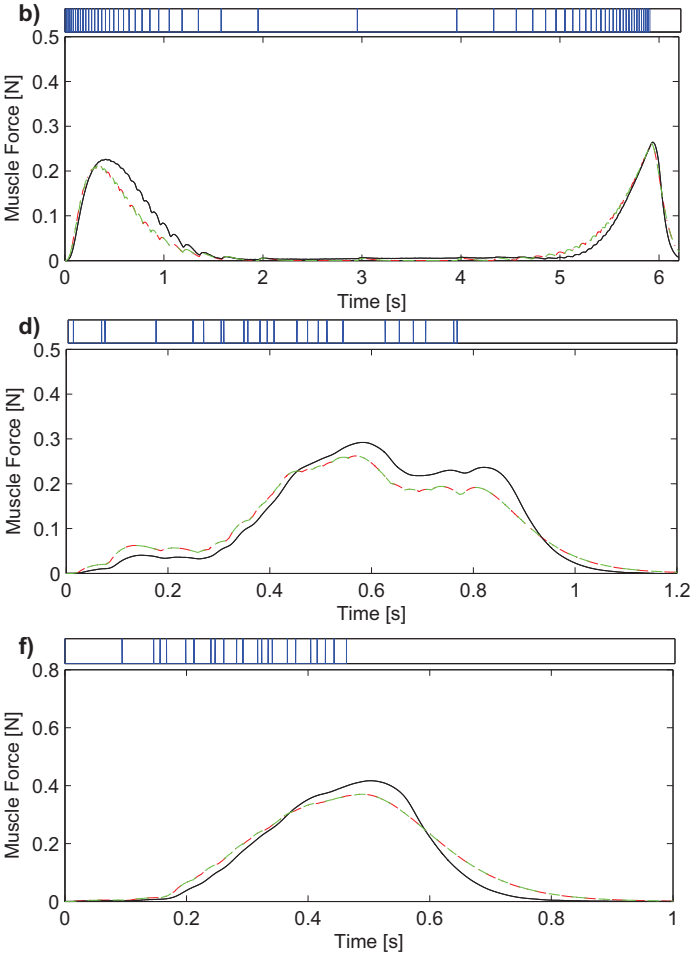

Fig. 8 Force response (-) and fit using the Adapted $\left(--_{-}\right)$and Simplified Adapted $\left({ }_{-}-_{-}\right)$models forF locust LSc. The pulses occur at times indicated in the bar above each figure. a,b) The response to general NCFTs, c-f) response to physiological SETi type inputs. 

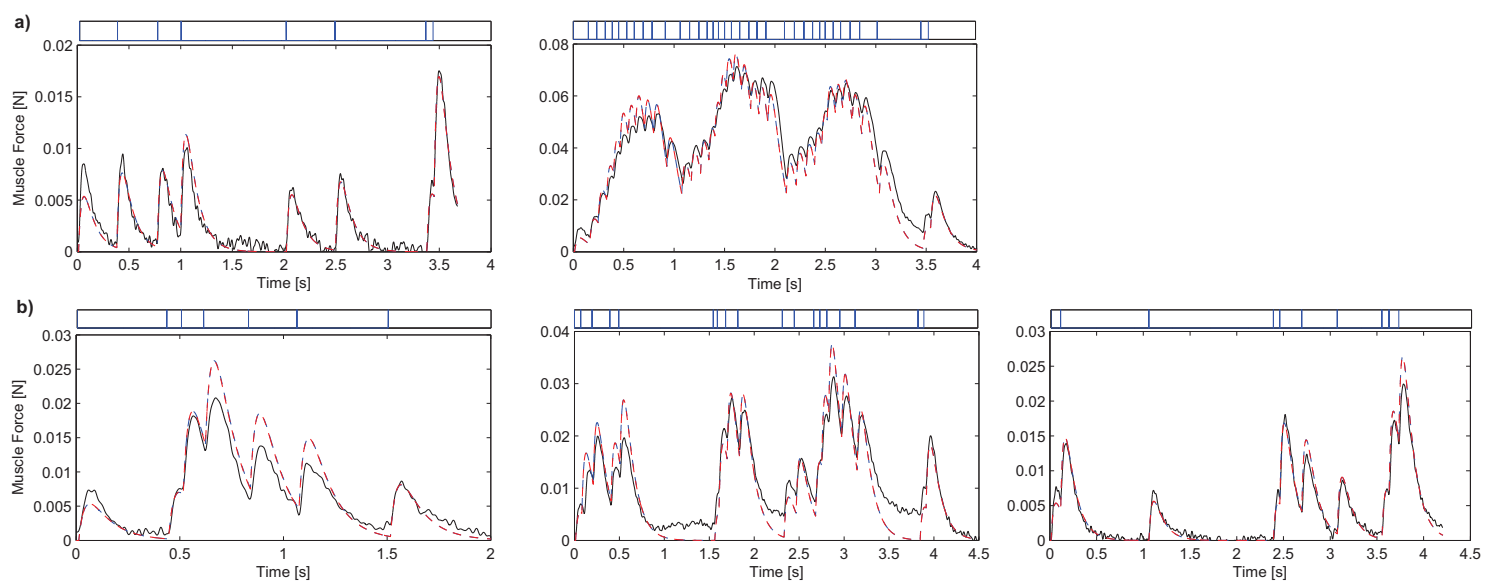

Fig. 9 Modelled fit using Simplified Adapted (- - ) and Adapted (- - ) models to measured voluntary activity (-). The times at which pulses occur are estimated from recorded neural activity of nerve $N .3 b$. and indicated in the bar above each figure. a) Fit to training data, b) fit to test data. Note that the modelled force responses are very similar, so traces overlay each other.

slow fibers generally saturate at a lower frequency than those of fast fibers (Mrowczynski et al., 2006). This difference is attributed to the structural specialisations of the slow fibers innervated by SETi, but not FETi Hoyle (1978). These fibers have a slowly relaxing core that is only activated during prolonged activation. The model has been shown to be capable of modelling both force responses that saturate at very high frequencies (SETi) and those that saturate at lower frequencies (FETi) by estimating different sets of model parameters for each case. Therefore, it should also be able to capture the dynamics of other muscles that saturate at different frequencies.

The responses to physiological SETi type inputs are similar, with all contractions lasting around $1 \mathrm{~s}$ with maximum forces of the order of $0.4 \mathrm{~N}$. In the stick insect extensor tibia muscle, bursts with approximately the same spike numbers and overall frequencies result in contractions that have very similar rises, even if the bursts have differing spike patterns (Hooper et al., 2007a). In other words variable inputs can produce similar outputs. A similar effect is seen in the physiological data, however, in many of the other recordings the average spike frequencies differ and so the resultant behaviour differs.

\subsection{Force Model}

In response to SETi stimulation the maximum measured forces (where multiple pulses sum) are about 60 times larger than the maximum force reached in response to a single stimulus. This is a much increased range than that of the response to FETi stimulation where the maximum measured forces were about 10 times larger than the maximum force response to a single stimulus. Attempting to model such a range of behaviour with a model developed for a different neuron provides a very good test of the model's capability to describe the response to a range of inputs, and potentially of a range of different muscles. Our model fits the SETi response well with errors of around $2 \%$. The errors in fit to the FETi response are consistently smaller, however the response to SETi stimulation is still well modelled.

The main discrepancies between the model predictions and actual force responses were in the fits to low-mid frequency CFT input, where the force was predicted to rise too quickly. This may be due to the model not capturing all the important mechanisms in the response to SETi stimulation. Hoyle (1978) proposes that calcium only reaches the core myofilaments during a relative long period of stimulation, that this core is slowly relaxing, and that during a twitch only the periphery of the fibres is recruited whereas during tetanus the core is progressively recruited. A model in which the core was progressively recruited (with time) could potentially result in the early rise in contraction being steadier and provide a better fit to data in response to low-mid frequency CFT inputs. Such a mechanism was included in our model to describe CFT stimulation by allowing the model parameters to change in a linear way with time since the first pulse. However, this did not improve fits and a good fit over the initial force rise in lower frequency CFTs resulted in the relaxation times being too great for high frequency CFTs (this indicates that the actual mechanism is more complicated than that proposed, it is likely to involve non-linearities, and perhaps other factors). Furthermore, this complicated the simple models and using the time after the 
first pulse in a contraction in the model equations does not provide a good general model for NCFT stimulation.

Although the models show some discrepancies in the fit to low-mid frequency CFT data they still provide a good model of the SETi response. For the low-mid frequency inputs for which the models do not predict the behaviour well the overall forces are low and so the absolute differences in the predicted and actual forces are still very small. Furthermore, although the frequency ranges are within those expected during natural SETi activity, a constant frequency input is an unrealistic input and the models are much better at describing the response to more physiologically relevant inputs, which are more applicable and relevant inputs. The models are able to predict the response to physiological SETi type inputs consistently across the ensemble of locusts and capture the dynamics of the behaviour well. In further support of the models, measured voluntary movements are very well reproduced when using the Simplified Adapted model (as seen in Fig. 9).

With the exception of one locust (LSa) the errors in fit to the Adapted model are little better than those when fitting to the Simplified Adapted model. The small improvement in errors when $\tau_{2}$ is included in the model and the fact the the value of $\tau_{2}$ varies between being positive and negative suggests that the parameter $\tau_{2}$ may not be required to model the SETi behaviour. The parameter $\tau_{2}$ modifies the time constant of equation (4), it is multiplied by the term $x(t)$ and added to the time constant $\tau_{1}$. In the case of the FETi response the value of $\tau_{2}$ is consistently positive, hence it acts to increase the time constant with increasing input, until saturation is reached. As the sign of $\tau_{2}$ is inconsistent in the response to SETi stimulation any biophysical interpretation of what $\tau_{2}$ represents is inconsistent as in some cases the time constant is increased with input, and in others decreased. Therefore, the Simplified Adapted model (equivalent to the Adapted model with $\tau_{2}=0$ ) may provide a better description of the SETi response, and in any event involves one fewer parameter, and so is a more parsimonious model. This is further supported by the fact that in most cases the force responses predicted by the Adapted and Simplified Adapted model are almost identical, but the Adapted model has one more parameter.

\subsection{Comparison with response to FETi stimulation}

The response of ETi to stimulation of the SETi motor neuron considered in this study can be compared to stimulation of the the fast counterpart, FETi, which was investigated in previous work (Wilson et al., 2010, 2011). Each fiber type is contained within the same ETi muscle, as described in Hoyle (1978). Stimulation of FETi produces twitches of large magnitude that, in response to CFT stimulation, sum to reach a steady state within a few spikes $(\approx 10)$. This all-or-nothing response is characteristic of the behaviour of vertebrate muscles (Hoyle, 1978). In contrast, stimulation of SETi produces very small twitches, approximately 100 times smaller in magnitude than the response to FETi, that sum over a large number of pulses. In the presented results, in response to CFT stimulation of SETi a steady state force was often not reached after 20 input pulses and Hooper et al. (2007b) report that slow muscles can take hundreds of spikes to achieve steady state. Further differences exist between the contraction times of the muscles, with the time course of contraction in response to SETi stimulation being around 1.5 times that in response to FETi stimulation. The contractile properties of the slow and fast fibers differ as they are responsible for different types of movements and so the physiology of their fibers and contractile mechanisms have evolved differences. Slow fibers have less Sarcoplasmic Reticulum (Klowden, 2002), and 0.3-0.5 times the number of $\mathrm{SR} \mathrm{Ca}^{2+}$ pump molecules (Baylor and Hollingworth, 2003). This means that calcium remains in the Sarcoplasm longer so that they have longer rates of relaxation. Furthermore, slow fibers have longer sarcomeres and a 6:1 ratio of actin:mysosin, compared to $3: 1$ in fast fibres, which may be in part responsible for the reduced force produced in comparison to fast fibres when the slow fibers contract.

We previously postulated the biophysical mechanisms that each model equation may describe (Wilson et al., 2011), suggesting that the quantity $C_{N}$ represents the calcium concentration in muscle filaments, with the time constant $\tau_{c}$ describing the time constant for calcium release from the SR. The last equation (Eq. (6)) was speculated to describe the rate-determining step in the formation of a cross-bridge between thick and thin filaments, with the non-linearity in magnitude accounting for the fact that the binding of calcium ions to troponin is a saturable non-linear reaction, with saturation occurring due to the limited number of binding sites. By comparing the response of each stage in the model for both FETi and SETi stimulation, the ability of the model to describe the expected differences, as related to the underlying physiology and structure, between the fiber types provides a test of how likely it is that these are the mechanisms described by each equation. Figure 10 shows the response at each stage in the Simplified Adapted model for a $13 \mathrm{~Hz}$ CFT input using the average estimated parameters for both FETi and SETi stimulation.

From Figure 10a it is evident that the time course of calcium decay is similar but slightly increased in the response to SETi. This is consistent with slow fibers having fewer pump molecules, resulting in calcium remaining in the SR longer. Rather than $C_{N}$ representing the absolute value of 

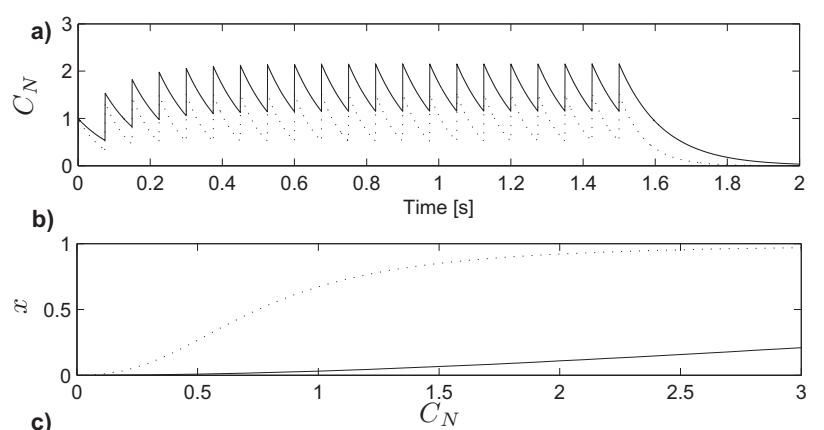

c)

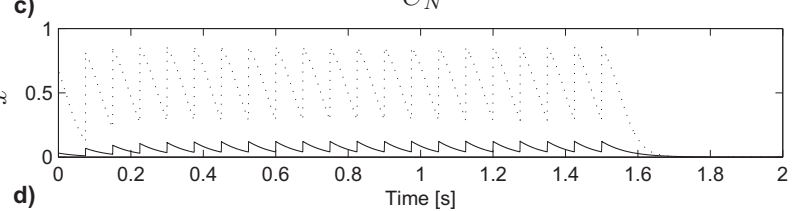

d)

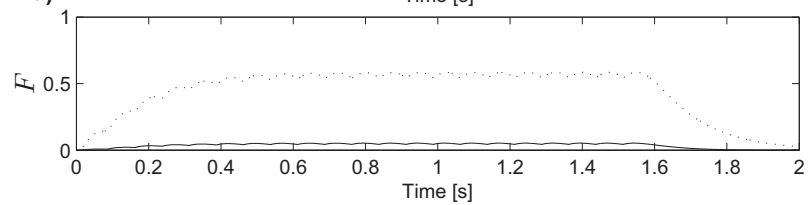

Fig. 10 Comparison of the response at each stage in the model for average parameters estimated in response to FETi $(-)$ and SETi $(\cdots)$ stimulation with a $13 \mathrm{~Hz}$ CFT input. a) total $C_{N}$, b) variation in $x$ with $\left.C_{N}, \mathrm{c}\right)$ total $x, \mathrm{~d}$ ) overall force.

calcium concentration in muscle filaments, it is more likely that it is a relative quantity that describes the time course of the calcium decay, especially considering that Eq. (5) contains no scaling factor, just a time constant of decay. One would expect the absolute calcium concentration to be higher in response to FETi stimulation due to the more extensive SR (Klowden, 2002) and the fact that fast fibres contain about 2.5 times the number of $\mathrm{Ca}^{2+}$ release channels of the SR in comparison to slow fibres (Baylor and Hollingworth, 2003). This supports the theory that $C_{N}$ is a relative quantity that represents the time course of calcium decay and gives the relative amount of calcium in muscle filaments for each particular fibre, so one can not draw comparisons between the magnitudes of the two values of $C_{N}$ at this stage. The amount of available calcium to form crossbridges $(x(t))$ (Fig. 10c) is predicted by the model to be much greater in response to FETi stimulation, as expected. Saturation occurs much later in the SETi response, shown by the fact that the non-linearity is still a long way from the maximum of 1 (Fig. 10c). This correlates with the fact that a steady state force is reached much faster in response to FETi stimulation.

These biomechanical processes are suggested rate-limiting steps that the model describes. Without monitoring calcium concentrations directly they cannot be verified. However, the Simplified Adapted model provides a good description of the expected behaviour of both fast and slow fibers.

The advantage of fitting models to data is that changes in model parameters can be investigated and interpreted, thus providing some quantification of the change in behaviour. The differences between the responses of the two fibre types can be quantified by both the differences in twitch characteristics and by the differences in parameter estimates (given in Tab 3). Different sets of locusts were used to estimate the SETi and FETi parameters, hence this discussion of parameter differences focuses on broad average changes between the two stimulation types. The parameter that varies the most between the FETi and SETi response is $k$, being an order of magnitude larger in the fit to SETi data. The value of $k$ determines the shape of the nonlinear function $x(t)$. A larger value of $k$ corresponds to saturation in the force level occurring at a larger input value which is seen to be the case in the SETi data. A comparison of the nonlinear functions is provided in Figure 10b, the main differences between the lines being due to the differences in the parameter $k$. The parameter $A$ is a gain, the value of $A$ estimated for the SETi response is consistently larger than that estimated for the FETi response. However, the magnitude of the FETi response is much larger, the majority of the difference in force levels is hence accounted for in the modelled response by the difference in $k$, as opposed to $A$. The parameter $\tau_{c}$ is on average 1.7 times greater for the fit to SETi data. This corresponds well with the increased contraction time of the SETi response, the other time constant, $\tau_{1}$, being slightly smaller for the SETi estimate. There are slight differences in the estimate of $m$, however these differences are similar to the differences observed across different locusts for the SETi response (see Table 3). Thus the differences may simply be due to the different sets of locusts used to estimate the muscle parameters in response to each type of stimulation. Previous studies (Bernotas et al., 1986; Bobet and Stein, 1998) that have fitted models to both the cat Soleus (a purely slow muscle) and Plantaris (a fast, fatigable muscle composed of mixed fibers), also report different parameter values for the fit to the different muscle types. In the model of Bobet and Stein (1998), which has a very similar form to the Simplified Adapted model (see Wilson et al. (2011)) for further comparisons of models), the rate constants and gain were greater ( 2-3 times) for the Plantaris compared to the Soleus, while the other parameters did not vary much.

\section{Conclusions}

In this study the isometric response of the locust hind leg extensor muscle to SETi stimulation was investigated, providing more data on the relatively poorly understood mechanical response to SETi stimulation. The measured force 
response was compared to the response of ETi to FETi stimulation, and the responses seen to differ significantly. Despite the significant differences in the force traces, a model developed to describe the response to FETi stimulation also provided a reasonable description of the SETi response if the parameters took different values. The presented model is thus able to describe the response to different neural inputs, across a wide range of different output forces, and hence provides a potentially generally applicable model of muscle response.

Acknowledgements We are grateful to the BBSRC and EPSRC for support for this study.

\section{References}

Ahn, A. N. and R. J. Full (2002). A motor and a brake: two leg extensor muscles acting at the same joint manage energy differently in a running insect. J Exp Biol 205, 379-89.

Alexander, R. M. (2003). Modelling approaches in biomechanics. Philos Trans R Soc Lond B Biol Sci 358, 142935.

Baylor, S. M. and S. Hollingworth (2003). Sarcoplasmic reticulum calcium release compared in slow-twitch and fast-twitch fibres of mouse muscle. J Physiol 551, 125138.

Bennet-Clark, H. C. (1975). The energetics of the jump of the locust schistocerca gregaria. J Exp Biol 63, 53-83.

Bernotas, L. A., P. E. Crago, and H. J. Chizeck (1986). A discrete-time model of electrically stimulated muscle. IEEE Trans Biomed Eng 33, 829-838.

Bobet, J. and R. B. Stein (1998). A simple model of force generation by skeletal muscle during dynamic isometric contractions. IEEE Trans Biomed Eng 45, 1010-6.

Burns, M. D. and P. N. R. Usherwood (1979). Control of walking in orthoptera .II. motor neuron activity in normal free-walking animals. J Exp Biol 79, 69-98.

Burrows, M. and G. A. Horridge (1974). The organization of inputs to motoneurons of the locust metathoracic leg. Philos Trans R Soc Lond B Biol Sci 269, 49-94.

Burrows, M. (1996). The Neurobiology of an Insect Brain. Oxford University Press, USA.

Cavanagh, P. and P. Komi (1979). Electromechanical delay in human skeletal muscle under concentric and eccentric contractions. Eur J Appl Physiol O 42, 159-163.

Dickinson, M. H., C. T. Farley, R. J. Full, M. A. R. Koehl, R. Kram, and S. Lehman (2000). How animals move: An integrative view. Science 288, 100-106.

Ding, J., A. S. Wexler, and S. A. Binder-Macleod (2002). A mathematical model that predicts the force-frequency relationship of human skeletal muscle. Muscle Nerve 26, 477-85.
Enoka, R. M. (2008). Neuromechanics of Human Movement, Fourth Edition. Sheridan Books, USA.

Frey Law, L. A. and R. K. Shields (2005). Mathematical models use varying parameter strategies to represent paralyzed muscle force properties: a sensitivity analysis. $J$ Neuroeng Rehabil 2, 18.

Guschlbauer, C., H. Scharstein, and A. Buschges (2007). The extensor tibiae muscle of the stick insect: biomechanical properties of an insect walking leg muscle. $J$ Exp Biol 210, 1092-108.

Heitler, W. J. (1977). Locust jump III. structural specializations of meta-thoracic tibiae. J Exp Biol 67, 29-36.

Hooper, S. L., C. Guschlbauer, G. von Uckermann, and A. Buschges (2007a). Different motor neuron spike patterns produce contractions with very similar rises in graded slow muscles. J Neurophysiol 97, 1428-1444.

Hooper, S. L., C. Guschlbauer, G. von Uckermann, and A. Buschges (2007b). Slow temporal filtering may largely explain the transformation of stick insect (carausius morosus) extensor motor neuron activity into muscle movement. J Neurophysiol 98, 1718-1732.

Hoyle, G. (1955a). The anatomy and innervation of locust skeletal muscle. Proc R Soc Lond B Biol Sci 143, 281-92.

Hoyle, G. (1955b). Neuromuscular mechanisms of a locust skeletal muscle. Proc R Soc Lond B Biol Sci 143, 343367.

Hoyle, G. (1978). Distributions of nerve and muscle fibre types in locust jumping muscle. J Exp Biol 73, 205-33.

Klowden, M. J. (Ed.) (2002). Physiological Systems in Insects. Academic Press.

Mrowczynski, W., J. Celichowski, and P. Krutki (2006). Interspecies differences in the force-frequency relationship of the medial gastrocnemius motor units. J Physiol Pharmacol 57, 491-501.

Newland, P. L. and Y. Kondoh (1997). Dynamics of neurons controlling movements of a locust hind leg III. extensor tibiae motor neurons. J Neurophysiol 77, 3297-3310.

The MathWorks Inc (2001). Curve Fitting Toolbox User's Guide. The MathWorks, Inc, Natick.

Usherwood, P. N. and H. I. Runion (1970). Analysis of mechanical responses of metathoracic extensor tibiae muscles of free-walking locusts. J Exp Biol 52, 39-58.

Usherwood, P. R. N. (Ed.) (1975). Insect Muscle. Academic Press Inc. Ltd.

Wilson, E., E. Rustighi, B. R. Mace, and P. L. Newland (2010). Isometric force generated by locust skeletal muscle: responses to single stimuli. Biol Cybern 102, 503511.

Wilson, E., E. Rustighi, B. R. Mace, and P. L. Newland (2011). Modelling the isometric force response to multiple pulse stimuli in locust skeletal muscle. Biol Cybern 104, 121-136. 
Wilson, E., E. Rustighi, P. L. Newland, and B. R. Mace (2011). A comparison of models of the isometric force of locust skeletal muscle in response to pulse train inputs. Biomech Model Mechanobiol 11, 519-532.

Wilson, E. (2010). Force Response of Locust Skeletal Muscle. $\mathrm{Ph}$. D. thesis, University of Southampton, Institute of Sound and Vibration Research.

Winters, J. M. (1995). How detailed should muscle models be to understand multijoint movement coordination. Hum Movement Sci 14, 401-442.

Zakotnik, J., T. Matheson, and V. Durr (2006). Cocontraction and passive forces facilitate load compensation of aimed limb movements. J Neurosci 26, 49955007. 